\title{
Reguladores de crescimento na frutificação efetiva e qualidade de frutos partenocárpicos de atemoia 'Gefner'
}

\author{
Marlon Cristian Toledo Pereira(1), Jonathan Henry Crane(2), Silvia Nietsche(1), \\ Wanda Montas $^{(2)}$ e Márcio Adriano Santos ${ }^{(1)}$
}

\begin{abstract}
(1)Universidade Estadual de Montes Claros, Departamento de Ciências Agrárias, Caixa Postal 91, CEP 39440-000 Janaúba, MG, Brasil. E-mail: marlon.pereira@unimontes.br, silvia.nietsche@unimontes.br, marcio.santos@ifnmg.edu.br (2)University of Florida, Institute of Food and Agricultural Sciences, Tropical Research \& Education Center, 18905 SW 280 ${ }^{\text {th }}$ St., 33031-3314 Homestead, FL, USA. E-mail: jhcr@ufl.edu, wmontas@ufl.edu
\end{abstract}

Resumo - O objetivo deste trabalho foi avaliar os efeitos de reguladores de crescimento vegetal sobre a frutificação efetiva e a qualidade de frutos partenocárpicos de atemoia 'Gefner'. O experimento foi realizado em pomar de atemoia 'Gefner', em Homestead, Flórida, EUA. Utilizou-se um delineamento experimental de blocos ao acaso, com quatro tratamentos, cinco repetições e seis flores por parcela. Os tratamentos foram compostos por: T1, ácido naftaleno acético a $450 \mathrm{mg} \mathrm{L}^{-1}$ e ácido giberélico a $1.000 \mathrm{mg} \mathrm{L}^{-1}$; T2, promalina a $100 \mathrm{mg} \mathrm{L}^{-1}$ e ácido giberélico a $1.000 \mathrm{mg} \mathrm{L}^{-1}$; T3, ácido giberélico a $1.000 \mathrm{mg} \mathrm{L}^{-1}$; e T4, polinização artificial (testemunha). Foram avaliados a frutificação efetiva, o crescimento e a qualidade dos frutos. A aplicação dos ácidos naftaleno acético e giberélico a flores de atemoia 'Gefner' proporcionou elevada frutificação efetiva, semelhante à da polinização artificial. A aplicação do ácido giberélico, associado ou não ao ácido naftaleno ou à promalina, resulta na produção de frutos de atemoia 'Gefner' mais tardios e menores do que os oriundos da polinização artificial, mas com boa proporção de polpa, excelente teor de sólidos solúveis e, principalmente, frutos sem sementes (partenocárpicos).

Termos para indexação: Annona cherimola, Annona squamosa, auxina, frutos sem sementes, giberelina, pegamento de fruto.

\section{Growth regulators on fruit set and quality of parthenocarpic fruit in atemoya 'Gefner'}

\begin{abstract}
The objective of this work was to evaluate the effects of plant growth regulators on the effective fruit set and quality of atemoya 'Gefner' parthenocarpic fruit. The experiment was carried out in an orchard of atemoya 'Gefner' in Homestead, Florida, USA. A randomized complete block design was carried out with four treatments, five replicates, and six flowers per plot. Treatments were composed of: T1, $450 \mathrm{mg} \mathrm{L}^{-1}$ naphthaleneacetic acid and $1,000 \mathrm{mg} \mathrm{L}^{-1}$ gibberelic acid; T2, $100 \mathrm{mg} \mathrm{L}^{-1}$ promalin and 1,000 $\mathrm{mg} \mathrm{L}^{-1}$ gibberelic acid; T3, 1,000 $\mathrm{mg} \mathrm{L}^{-1}$ gibberelic acid; and T4, artificial pollination (control). Effective fruit set, and fruit growth and quality were evaluated. The application of naphthaleneacetic and gibberelic acids on flowers of atemoya 'Gefner' provided high fruit set, similar to that of artificial pollination. Gibberelic acid application, associated or not with naphthaleneacetic acid or promalin, results in later and smaller atemoya 'Gefner' fruit than those obtained by artificial pollination, but with good proportion of pulp, excellent soluble solids content and, mainly seedless fruit (parthenocarpic fruit).
\end{abstract}

Index terms: Annona cherimola, Annona squamosa, auxin, seedless fruit, gibberellin, fruit set.

\section{Introdução}

O desenvolvimento de cultivares sem sementes tem atraído a atenção de produtores, que destacam a facilidade para o consumo in natura ou processado e o valor agregado do produto final, como algumas vantagens advindas desta nova característica (Pandolfini, 2009; Lora et al., 2011). De acordo com Maestrelli et al. (2003), a ausência de sementes está associada ao aumento da qualidade dos frutos. Esses mesmos autores reportam que a presença de sementes muito duras, o seu gosto desagradável e a capacidade de produção de substâncias que aceleram o processo de deterioração dos frutos são fatores que afetam a qualidade dos frutos.

Frutos sem sementes podem ser decorrentes de mutações, como em tomateiro e pinheira, ou de produção por meio de métodos tradicionais ou biotecnológicos 
aplicados ao melhoramento, como em uva, citros e melancia (Kihara, 1951; Gourget et al., 2005; Lora et al., 2011). Uma alternativa viável à produção de frutos partenocárpicos consiste no tratamento das flores com uso de reguladores de crescimento vegetal, aplicados antes da polinização. Entre os reguladores, as auxinas, giberelinas e citocininas ou a mistura destas tem sido eficiente no pegamento e no desenvolvimento de frutos (Schwabe \& Mills, 1981).

Auxinas e giberelinas são grupos de fitormônios que estão associados aos processos de polinização, fertilização e desenvolvimento dos frutos (Nitsch, 1952). Em estudos realizados em ervilha e tomate, tem-se observado efeito sinérgico entre esses fitorreguladores, o que indica que a presença de ambos na regulação do desenvolvimento dos frutos é requisito fundamental (Ozga \& Reinecke, 1999; Jong et al., 2009). Trabalhos com a cultura do tomateiro mostram que a aplicação exógena de auxinas a flores não polinizadas induzem o pegamento e o crescimento de frutos (Srivastava \& Handa, 2005; Dorcey et al., 2009). Da mesma maneira, as giberelinas, como o $\mathrm{GA}_{3}$, também desempenham papel fundamental no processo de pegamento e crescimento do fruto, bem como no desenvolvimento das sementes (Serrani et al., 2007; Casanova et al., 2009; Jong et al., 2009). De acordo com Saavedra (1979), tanto o ácido naftaleno acético (ANA) como as giberelinas proporcionaram a produção de frutos sem sementes em cherimoia.

A atemoia é um híbrido interespecífico entre a cherimoia (Annona cherimola Mill.) e a pinha (Annona squamosa L.), cujo crescimento vem sendo observado em diversos países de clima tropical e subtropical, como Austrália, Brasil, Israel e os estados da Flórida e do Havaí nos Estados Unidos da América (Crane et al., 2005). A atemoia apresenta alta produtividade em cultivo irrigado, com possibilidade de duas safras ao ano, além da qualidade dos frutos e bons preços no mercado, e é excelente opção para a diversificação da produção agrícola (Pereira et al., 2011; Pereira \& Kavati, 2011).

Apesar do crescente interesse pelo cultivo deste híbrido, observam-se problemas associados à baixa viabilidade dos grãos de pólen, alto custo da polinização artificial, bem como o elevado número de sementes por fruto; tais problemas precisam ser solucionados com o objetivo reduzir o custo de produção e popularizar a fruta junto ao mercado consumidor (Melo et al., 2002; Bettiol Neto et al., 2009; Mendes et al., 2012).

O objetivo deste trabalho foi avaliar os efeitos de reguladores de crescimento vegetal sobre a frutificação efetiva e a qualidade de frutos partenocárpicos de atemoia 'Gefner'.

\section{Material e Métodos}

O trabalho foi realizado em pomar experimental de atemoia 'Gefner' (Annona cherimola Mill. x Annona squamosa L.), com plantas de 12 anos de idade, conduzidas em espaçamento $6 \times 4 \mathrm{~m}$, no Tropical Research \& Education Center $\left(25^{\circ} 50^{\prime} \mathrm{N}, 80^{\circ} 50^{\prime} \mathrm{W}\right.$, à altitude de 3,8 m), Universidade da Flórida, Município de Homestead, EUA. O sistema de irrigação utilizado foi o de aspersão convencional, duas vezes por semana, duas horas por dia, tendo-se considerado a demanda de $1.000 \mathrm{~mm}$ anuais.

Com relação aos tratos culturais, as plantas foram podadas mecanicamente e manualmente em março de 2011. As plantas daninhas foram controladas com roçadeira mecânica a cada dois meses. Na projeção da copa, utilizou-se cobertura morta de casca de pinus (Pinus elliottii). O controle da broca-das-sementes (Bephratelloides pomorum, Fabricius, 1808) foi realizado com pulverizações mensais de julho a setembro de 2001. A adubação foi realizada de acordo com a análise de solo, tendo-se aplicado $160 \mathrm{~g}$ de $\mathrm{P}_{2} \mathrm{O}_{5}$ por planta na forma de MAP (fosfato monoamônico), em aplicação única, após a poda. O sulfato de amônio e o cloreto de potássio foram aplicados de forma parcelada em três vezes, a cada dois meses a partir de março de 2011, no total de $180 \mathrm{~g}$ de $\mathrm{N}$ por planta e $200 \mathrm{~g}$ de $\mathrm{K}_{2} \mathrm{O}$ por planta. Os micronutrientes foram aplicados via foliar em duas pulverizações, nos meses de junho e agosto.

O experimento foi instalado em 7/6/2011, com 10 plantas de atemoia 'Gefner' selecionadas e identificadas, tendo-se observado a uniformidade, o vigor e a sanidade. Utilizou-se o delineamento experimental de blocos ao acaso com quatro tratamentos, cinco repetições e seis flores por parcela, identificadas com fitas de lã colorida. Aplicaram-se todos os tratamentos a cada planta, com três flores por tratamento, no total de 12 flores por planta.

Os tratamentos utilizados foram: T1, ácido naftaleno acético (ANA) a $450 \mathrm{mg} \mathrm{L}^{-1}$ e ácido giberélico $\left(\mathrm{GA}_{3}\right)$

Pesq. agropec. bras., Brasília, v.49, n.4, p.281-289, abr. 2014

DOI: 10.1590/S0100-204X2014000400006 
a $1.000 \mathrm{mg} \mathrm{L}^{-1}$; $\mathrm{T} 2$, promalina a $100 \mathrm{mg} \mathrm{L}^{-1}$ e $\mathrm{GA}_{3}$ a $1.000 \mathrm{mg} \mathrm{L}^{-1} ; \mathrm{T} 3, \mathrm{GA}_{3}$ a $1.000 \mathrm{mg} \mathrm{L}^{-1}$; e T4, polinização artificial (testemunha). Os produtos comerciais utilizados foram: K-Salt Fruit Fix 200 (AMVAC Chemical Corporation, Los Angeles, CA, EUA) com 5,18\% de ANA; ProGibb (Valent BioSciences Corporation Technology Way, Libertyville, IL, USA) com $4 \%$ de $\mathrm{GA}_{3}$; e promalina (Valent BioSciences Corporation Technology Way, Libertyville, IL, USA) com 1,8\% de $\mathrm{GA}_{4,7}$. As soluções dos reguladores de crescimento foram preparadas com diluição dos produtos comerciais em água, com auxílio de balão volumétrico. As soluções foram aplicadas com uso de frascos tipo spray e direcionadas às flores durante a antese, ou seja, flores em estádio funcionalmente pistilado (pré-feminino a feminino).

Após o pegamento dos frutos, realizaram-se três aplicações dos tratamentos, direcionadas aos frutos aos 7, 21 e 35 dias após a antese (DAA). A polinização artificial foi realizada por meio de grãos de pólen de pinheiras, com auxílio de um pincel número seis.

Os efeitos dos tratamentos foram avaliados semanalmente, tendo-se observado pegamento dos frutos, frutificação efetiva, comprimento e diâmetro dos frutos. A colheita dos frutos foi realizada quando eles apresentavam afastamento dos carpelos, coloração verde-amarelada dos tecidos intercarpelares e cor da casca verde mais claro, que ocorreu a partir de 108 dias após a antese.

Os frutos de cada tratamento foram identificados, acondicionados em sacolas de papel e, posteriormente, em caixas de plástico, e conduzidos ao Laboratório de Pós-Colheita da Universidade da Flórida, em Homestead, EUA. Em seguida, avaliaram-se: a massa de matéria fresca, o comprimento, o diâmetro e o formato dos frutos, além do diâmetro do pedúnculo dos frutos. Determinou-se o formato dos frutos em percentagens, em que frutos totalmente irregulares receberam nota igual a $25 \%$; parcialmente irregulares, nota $50 \%$; regulares, com pouca deformação, nota $75 \%$; e totalmente regulares (fruto arredondado perfeito), nota $100 \%$. Os frutos foram mantidos no laboratório à temperatura constante de $25^{\circ} \mathrm{C}$, até o amadurecimento.

Por ocasião do amadurecimento, que ocorreu seis dias após a colheita, os frutos foram avaliados quanto à massa de matéria fresca, em balança digital modelo TR-6101 (Denver Instrument Bohemia, NY, EUA), e quanto à firmeza, com auxílio de penetrômetro manual Bishop FT 011 (QA Supplies, LLC, Norfolk, Virginia, U.S.A), com ponteira de $8 \mathrm{~mm}$ de diâmetro. Em seguida, realizou-se a separação da casca, polpa e sementes, para determinação de suas respectivas massas. Foi feita a contagem do número de sementes, quando encontradas, e uma alíquota de polpa dos frutos foi separada para a análise do teor de sólidos solúveis, com uso de refratômetro manual (Atago U.S.A., Bellevue, WA, U.S.A.).

O comprimento e o diâmetro dos frutos foram ajustados à equação logística $\left[y=a /\left(1+b e^{c x}\right)\right]$, em que: y é o comprimento ou o diâmetro dos frutos; a é o parâmetro assintótico que corresponde ao comprimento ou ao diâmetro do fruto à maturação; b é o parâmetro de locação, sem interpretação biológica; e é à base dos logaritmos neperianos; c é a taxa de expansão do crescimento; e x é o tempo em que o crescimento ocorre, dado em semanas após a antese. As taxas de crescimento foram obtidas pela derivada primeira da equação ajustada (Richards, 1969). As demais características avaliadas foram submetidas à análise de variância pelo programa estatístico Sisvar (Ferreira, 2008) e, quando significativas, foram submetidas ao teste de Tukey, a 5\% de probabilidade, para comparação das médias.

\section{Resultados e Discussão}

Até 63 dias após a antese (DAA), observaram-se $100 \%$ de pegamento dos frutos e frutificação efetiva com o uso da polinização artificial (Figura 1). Após este período e até 112 DAA, verificou-se pequena queda de frutos, com fixação de $97 \%$ dos frutos na colheita. Entre os tratamentos com reguladores, o $\mathrm{ANA}+\mathrm{GA}_{3}$ promoveu $100 \%$ de pegamento dos frutos até 21 DAA. Após este período, observou-se queda de frutos até os 77 DAA, com fixação final de $87 \%$ de frutos. Os tratamentos com promalina $+\mathrm{GA}_{3}$ e apenas $\mathrm{GA}_{3}$ proporcionaram $100 \%$ de pegamento até os 14 DAA. Após este período, constataram-se reduções de até $50 \%$ de frutificação efetiva ao final da avaliação.

Com os resultados do presente trabalho, temse a indicação de que a participação de ambos os reguladores de crescimento (auxinas e giberelinas) é fundamental no pegamento de frutos e na frutificação efetiva da atemoia, com resultados semelhantes aos da polinização artificial. Yonemoto et al. (2000), ao avaliar a fixação de frutos sem sementes em 11 cultivares de 
cherimoia, com uso de 1.600 ppm de $\mathrm{GA}_{3}$, observaram apenas quatro cultivares com pegamento de frutos superior a $80 \%$, após 77 dias após a aplicação do tratamento. Resultados semelhantes foram obtidos por Koura et al. (2004), quando avaliaram o uso de $\mathrm{GA}_{3}$ e o pegamento de frutos nas cultivares de cherimoia Big Sister e Suiho. Em estudo realizado com uso de $450 \mathrm{mg} \mathrm{L}^{-1}$ de ANA em atemoia 'Gefner', Mota Filho et al. (2012) verificaram a baixa eficiência deste regulador, com apenas $8 \%$ de pegamento de frutos, aos 148 dias após a primeira aplicação.

Em todos os tratamentos, houve incremento rápido do comprimento dos frutos, nas primeiras semanas, que atingiu o valor máximo por volta de 28 DAA, com taxa de crescimento de $14,6 \mathrm{~mm}$ por semana na polinização artificial, e média de $10,8 \mathrm{~mm}$ por semana para os tratamentos com reguladores de crescimento (Figura 2). A partir destes pontos máximos, houve decréscimo contínuo desta taxa, em todos os tratamentos, com crescimento lento dos frutos em valores inferiores a $1 \mathrm{~mm}$ por semana, a partir de 70 DAA, até a colheita. Comportamento similar foi observado para o crescimento em diâmetro dos frutos (Figura 3). O rápido crescimento inicial dos frutos mostra a importância do parcelamento das aplicações dos reguladores de crescimento, nesta fase inicial, que acompanha o mesmo comportamento dos frutos advindos de flores polinizadas. O emprego de produto a base de auxina, giberelina e citocinina, em videira 'Niagara Rosada', nas doses de 5 a $20 \mathrm{mg} \mathrm{L}^{-1}$, promoveu aumento linear no comprimento do cacho e aumento quadrático do pedicelo (Tecchio et al., 2006).

As aplicações de reguladores de crescimento resultaram na produção de frutos significativamente mais tardios, em comparação à polinização artificial, com até 18 dias a mais da antese à colheita (Tabela 1). Isto mostrou a importância da presença das sementes, nos frutos de atemoia 'Gefner', na precocidade de produção. Comportamento similar foi mantido até o amadurecimento dos frutos, que apresentaram ponto de consumo cerca de seis dias após a colheita.

Frutos oriundos da polinização artificial apresentaram maior massa de matéria fresca e massa fresca da polpa, em comparação aos frutos obtidos por meio de reguladores de crescimento (Tabelas 1 e 2). Em média, observou-se redução de $49 \%$ da massa de matéria fresca destes frutos, em relação aos frutos obtidos por meio da polinização artificial. No entanto, a perda de massa de matéria fresca da colheita ao amadurecimento foi maior nos frutos advindos da aplicação de reguladores de crescimento, possivelmente, em razão da presença

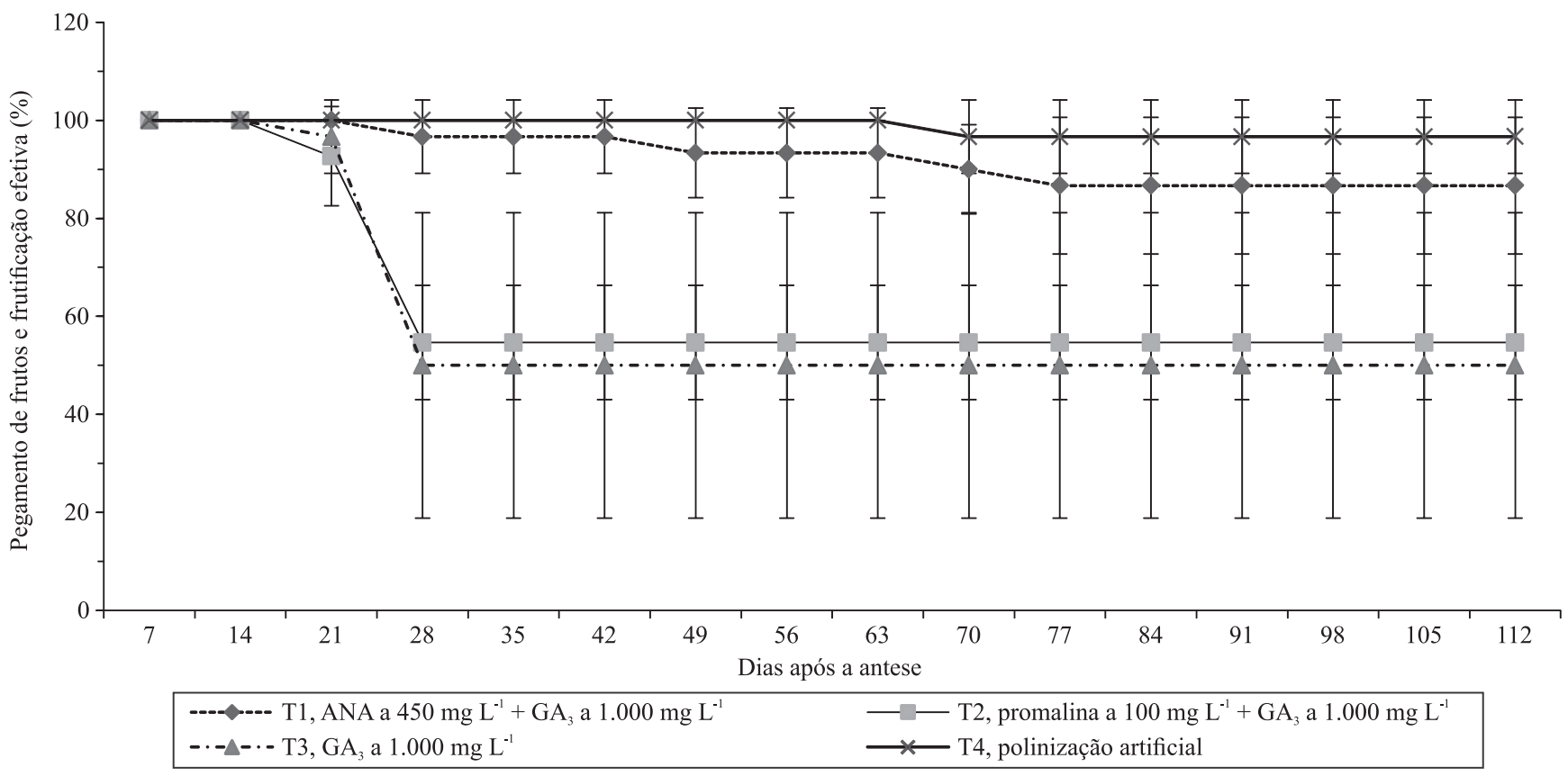

Figura 1. Percentagem de pegamento de frutos e frutificação efetiva de atemoia 'Gefner', após a aplicação dos reguladores de crescimento e da polinização artificial, em Homestead, Flórida, EUA. ANA, ácido naftaleno acético; GA 
das sementes nos frutos advindos de polinização artificial, o que confere maior consistência a estes frutos. Ao analisar os dados de massa de matéria fresca da polpa e da casca, constataram-se reduções médias de 63 e $26 \%$, respectivamente, nos frutos resultantes da polinização, em relação aos que receberam aplicação dos reguladores de crescimento. $\mathrm{O}$ comprimento e o diâmetro dos frutos apresentaram comportamento similar, ou seja, frutos provenientes da polinização artificial foram maiores do que os frutos obtidos por meio da aplicação de reguladores de crescimento, os quais não diferiram entre si (Tabela 2). De acordo com Pires et al. (2003), a aplicação do ácido giberélico a $5 \mathrm{mg} \mathrm{L}^{-1}$ proporcionou incremento na massa e na largura dos cachos, no comprimento e na largura das bagas, além de $78,7 \%$ de aumento na massa das bagas da uva 'Centennial Seedless'. Moreira et al. (2010) observaram que o ácido giberélico a $150 \mathrm{mg} \mathrm{L}^{-1}$ foi
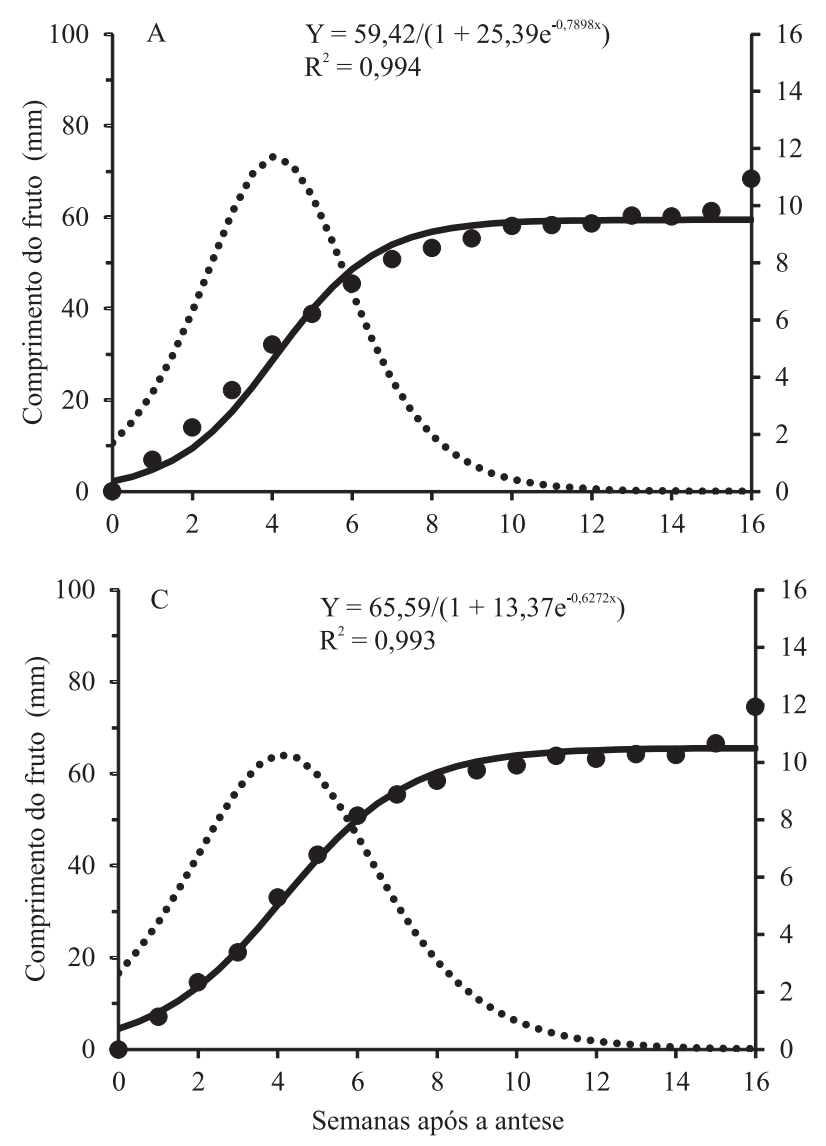

efetivo no aumento do comprimento e do diâmetro das bagas, bem como da massa dos cachos da videira 'Niagara Rosada'.

A aplicação exógena de reguladores de crescimento promoveu desenvolvimento normal da fase I em frutos de atemoia 'Gefner', isto é, os reguladores permitiram que ocorressem as atividades correlatas à polinização e de vingamento dos frutos. Entretanto, os problemas de redução das dimensões destes frutos foram observados nas fases II e III, caracterizadas pela intensa divisão e expansão celular. No desenvolvimento normal de frutos, os embriões e as sementes controlam a taxa e a manutenção da divisão celular, por meio da produção de hormônios vegetais como auxinas, giberelinas e citocininas. Além da produção dos hormônios, o número de óvulos fertilizados também afeta a taxa de divisão celular (Varga \& Bruinsma, 1986). No presente trabalho, provavelmente, as doses de reguladores de
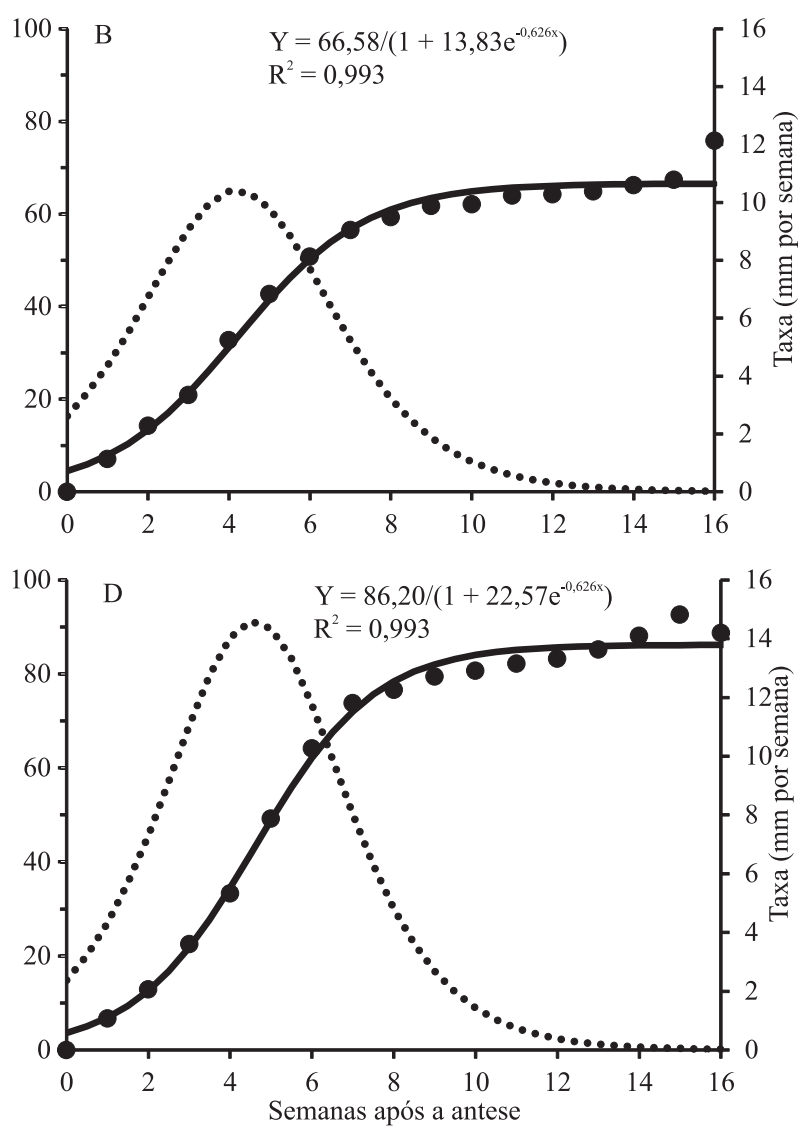

- Média Curva $\longrightarrow$ C.... Taxa

Figura 2. Curva e taxa de crescimento do comprimento dos frutos de atemoia 'Gefner', após a aplicação dos tratamentos: T1, ANA a $450 \mathrm{mg} \mathrm{L}-1+\mathrm{GA}_{3}$ a $1.000 \mathrm{mg} \mathrm{L}^{-1}(\mathrm{~A})$; T2, promalina a $100 \mathrm{mg} \mathrm{L}^{-1}+\mathrm{GA}_{3}$ a $1.000 \mathrm{mg} \mathrm{L}-1$ (B); T3, GA 3 a $1.000 \mathrm{mg}$

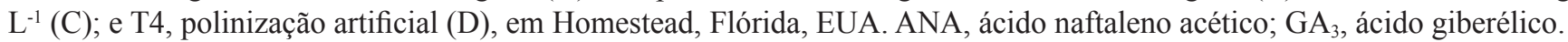


crescimento não foram suficientes para substituir a produção endógena dos hormônios de crescimento pelas sementes. De acordo com Jong et al. (2009), o crescimento de órgãos vegetais promovidos por giberelinas ocorre principalmente em razão do aumento do volume das células já existentes ou recentemente divididas, entretanto, normalmente, observa-se que frutos sem sementes apresentam tamanho inferior aos que possuem sementes.

Frutos originados da polinização artificial apresentaram, em média, 69 sementes por fruto (Tabela 2). A presença das sementes nos frutos de atemoia é fundamental para os processos de divisão e expansão celular dos frutos. A aplicação exógena dos reguladores de crescimento $\mathrm{GA}_{3}$, associado ou não ao ANA e à promalina, embora tenha permitido a produção de frutos sem sementes ou com traços de sementes, considerados paternocárpicos, proporcionou
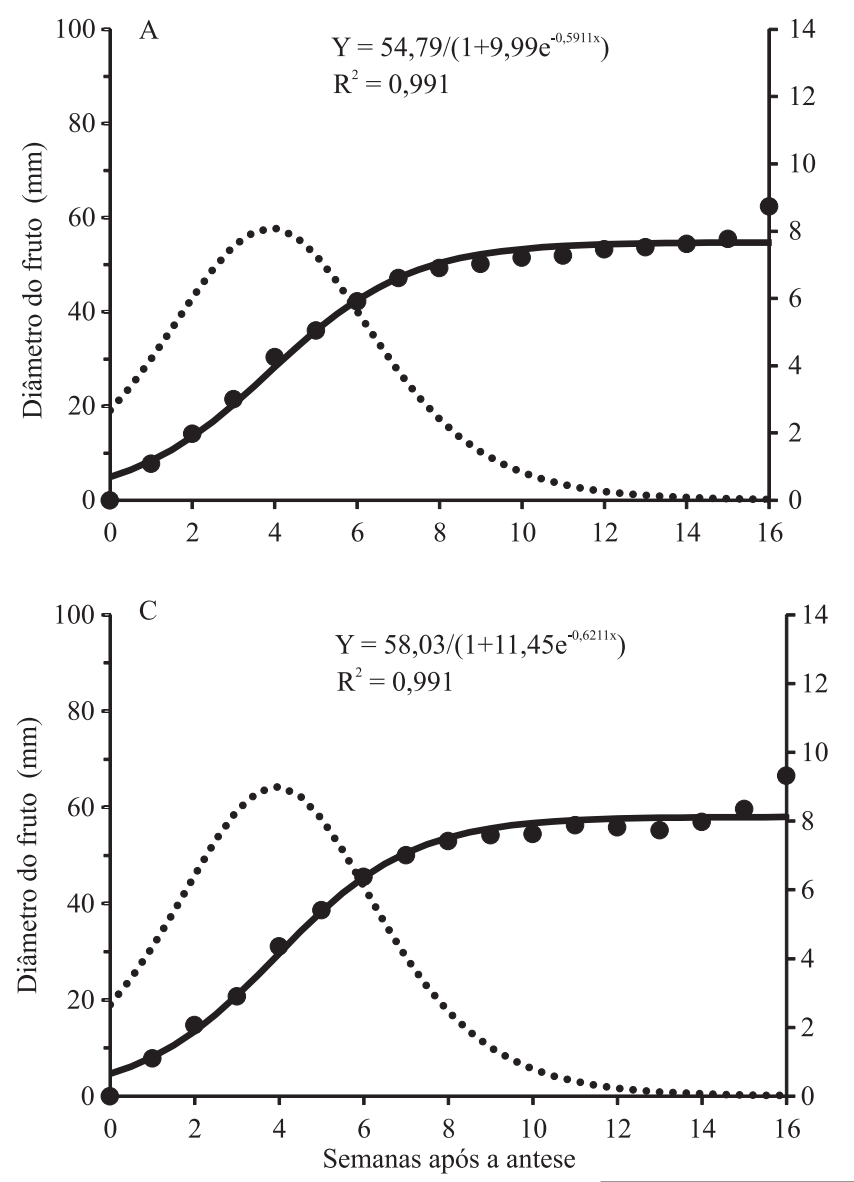

- Média Curva -...... Taxa

Figura 3. Curva e taxa de crescimento do diâmetro dos frutos de atemoia 'Gefner', após a aplicação dos tratamentos: T1, ANA a $450 \mathrm{mg} \mathrm{L}^{-1}+\mathrm{GA}_{3}$ a $1.000 \mathrm{mg} \mathrm{L}^{-1}$ (A); T2, promalina a $100 \mathrm{mg} \mathrm{L}^{-1}+\mathrm{GA}_{3}$ a $1.000 \mathrm{mg} \mathrm{L}^{-1}$ (B); T3, GA a $1.000 \mathrm{mg}$ $\mathrm{L}^{-1}$ (C); e T4, polinização artificial (D), em Homestead, Flórida, EUA. ANA, ácido naftaleno acético; GA 3 , ácido giberélico. também a produção de frutos menores. Estudos realizados com o uso de reguladores de crescimento da família Annonaceae, mostraram a capacidade das giberelinas no incremento do pegamento e na produção de frutos sem sementes, o que indica, por parte deste regulador de crescimento, uma substituição parcial da função exercida pelo grão de pólen, no momento da polinização e fertilização (Saavedra, 1979; Koura et al., 2004; Mota Filho et al., 2012).

Com o uso de $\mathrm{ANA}+\mathrm{GA}_{3}$ obteve-se maiores valores de sólidos solúveis do que nos demais tratamentos (Tabela 2). Entretanto, todos os tratamentos alcançaram valores elevados e dentro dos padrões comerciais, que variam entre 20 e $32^{\circ}$ Brix, o que comprova a excelente qualidade de todos os frutos produzidos.

A polinização artificial das flores de atemoia proporcionou a produção de frutos mais uniformes do
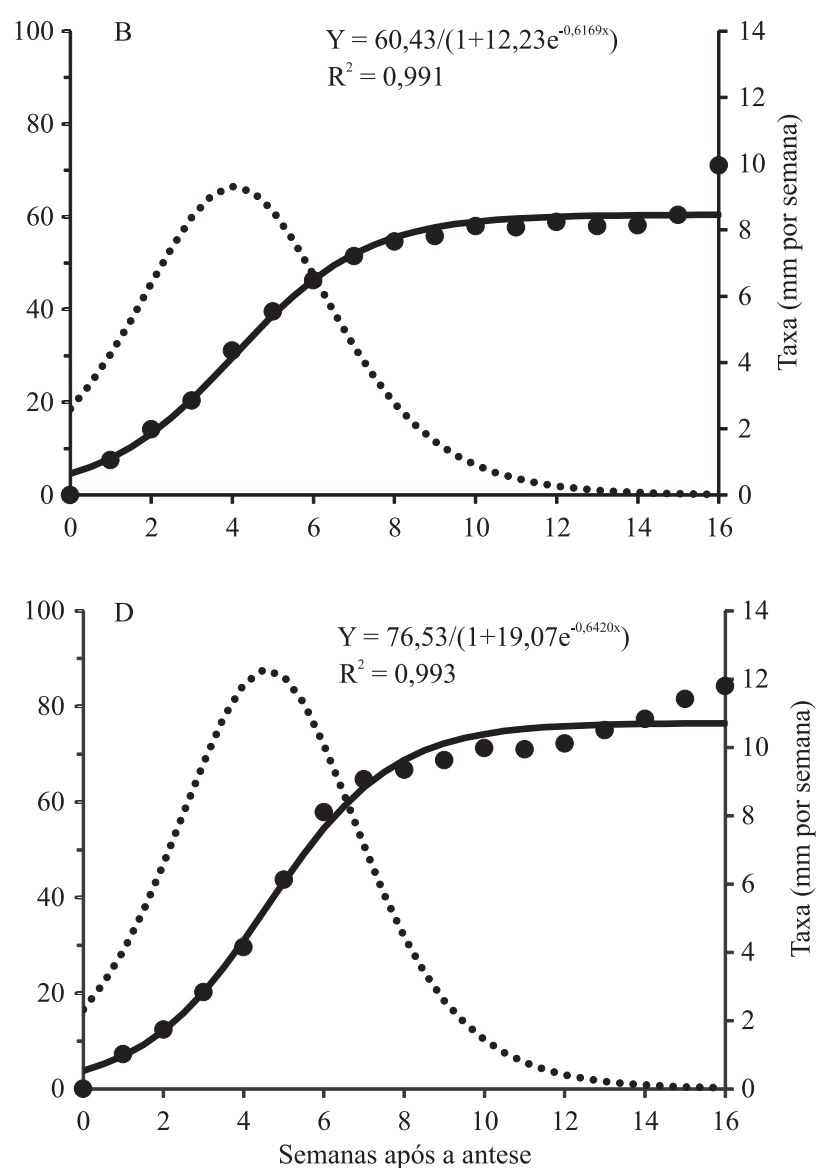
para a obtenção de frutos paternocárpicos, em espécies 
que os frutos provenientes da aplicação de reguladores de crescimento, os quais apresentaram formatos mais irregulares e com pouca deformação (Tabela 2). A irregularidade ocorre provavelmente em função da ausência de sementes e polpa, em algumas partes dos frutos, o que provoca a atrofia dos carpelos, com consequente alteração da forma natural (Pereira et al., 2003). Frutos de atemoia 'Gefner', sem sementes e

Tabela 1. Número de dias da antese à colheita (NDAC), número de dias da antese ao amadurecimento (NDAA), massa de matéria fresca do fruto na colheita (MFF), percentagem de perda de massa de matéria fresca da colheita ao amadurecimento (PMF), comprimento e diâmetro de frutos de atemoia, após a aplicação de reguladores de crescimento e polinização artificial, em Homestead, Flórida, EUA, 2011 ${ }^{(1)}$

\begin{tabular}{|c|c|c|c|c|c|c|}
\hline Tratamento & $\begin{array}{l}\text { NDAC } \\
\text { (dias) }\end{array}$ & $\begin{array}{c}\text { NDAA } \\
\text { (dias) }\end{array}$ & $\begin{array}{c}\text { MFF } \\
(\mathrm{g})\end{array}$ & $\begin{array}{c}\text { PMF } \\
(\%)\end{array}$ & $\begin{array}{c}\text { Comprimento } \\
\text { (mm) }\end{array}$ & $\begin{array}{c}\text { Diâmetro } \\
(\mathrm{mm})\end{array}$ \\
\hline $\mathrm{T} 1$, ANA a $450 \mathrm{mg} \mathrm{L}^{-1}+\mathrm{GA}_{3}$ a $1.000 \mathrm{mg} \mathrm{L}^{-1}$ & $126 \mathrm{a}$ & $132 \mathrm{a}$ & $135,7 \mathrm{~b}$ & $25,1 \mathrm{a}$ & $76,2 b$ & $68,5 b$ \\
\hline $\mathrm{T} 2$, promalina a $100 \mathrm{mg} \mathrm{L}^{-1}+\mathrm{GA}_{3}$ a $1.000 \mathrm{mg} \mathrm{L}^{-1}$ & $121 b$ & $127 b$ & $159,7 \mathrm{~b}$ & $20,3 b$ & $79,6 b$ & $72,5 b$ \\
\hline $\mathrm{T} 3, \mathrm{GA}_{3}$ a $1.000 \mathrm{mg} \mathrm{L}^{-1}$ & $123 \mathrm{ab}$ & $129 \mathrm{ab}$ & $141,9 b$ & $22,3 \mathrm{ab}$ & $77,5 b$ & $71,4 b$ \\
\hline T4, polinização artificial & $108 \mathrm{c}$ & $113 \mathrm{c}$ & $298,9 \mathrm{a}$ & $13,2 \mathrm{c}$ & $94,6 \mathrm{a}$ & $85,9 \mathrm{a}$ \\
\hline $\mathrm{CV}(\%)$ & 1,75 & 1,61 & 12,60 & 10,54 & 6,12 & 5,07 \\
\hline
\end{tabular}

${ }^{(1)}$ Médias seguidas de letras distintas, nas colunas, diferem pelo teste de Tukey, a 5\% de probabilidade. ANA, ácido naftaleno acético; GA 3 , ácido giberélico.

Tabela 2. Massa de matéria fresca da polpa (MFP), massa de matéria fresca da casca (MFC), massa de matéria fresca da semente (MFS), número de sementes (NS), teor de sólidos solúveis (SS), formato dos frutos (FORM) e firmeza dos frutos (FIRM) de atemoia, após a aplicação de reguladores de crescimento e da polinização artificial, em Homestead, Flórida, EUA, $2011^{(1)}$

\begin{tabular}{|c|c|c|c|c|c|c|c|}
\hline Tratamento & $\begin{array}{c}\text { MFP } \\
(\mathrm{g})\end{array}$ & $\begin{array}{c}\text { MFC } \\
(\mathrm{g})\end{array}$ & $\begin{array}{c}\text { MFS } \\
(\mathrm{g})\end{array}$ & $\begin{array}{l}\text { NS } \\
\text { (un) }\end{array}$ & $\begin{array}{c}\mathrm{SS} \\
\left({ }^{\circ} \text { Brix }\right)\end{array}$ & $\begin{array}{c}\text { FORM } \\
(\%)\end{array}$ & $\begin{array}{c}\text { FIRM } \\
(\mathrm{N})\end{array}$ \\
\hline T1, ANA a $450 \mathrm{mg} \mathrm{L}^{-1}+\mathrm{GA}_{3}$ a $1.000 \mathrm{mg} \mathrm{L}^{-1}$ & $52,2 b$ & $49,6 \mathrm{~b}$ & $0,1 \mathrm{~b}$ & $0,4 \mathrm{~b}$ & $31,1 \mathrm{a}$ & $63,7 \mathrm{~b}$ & $6,9 b$ \\
\hline $\mathrm{T} 2$, promalina a $100 \mathrm{mg} \mathrm{L}^{-1}+\mathrm{GA}_{3}$ a $1.000 \mathrm{mg} \mathrm{L}^{-1}$ & $65,4 b$ & $62,0 \mathrm{ab}$ & $0,0 \mathrm{~b}$ & $0,0 \mathrm{~b}$ & $28,5 b$ & $61,3 b$ & $8,4 \mathrm{~b}$ \\
\hline $\mathrm{T} 3, \mathrm{GA}_{3}$ a $1.000 \mathrm{mg} \mathrm{L}^{-1}$ & $54,7 \mathrm{~b}$ & $56,1 b$ & $0,2 b$ & $0,6 b$ & $29,1 b$ & $54,7 b$ & $9,2 b$ \\
\hline T4, polinização artificial & $156,2 \mathrm{a}$ & $75,7 \mathrm{a}$ & $27,9 \mathrm{a}$ & $69,2 \mathrm{a}$ & $28,9 b$ & $87,2 \mathrm{a}$ & $16,2 \mathrm{a}$ \\
\hline CV (\%) & 13,51 & 13,98 & 34,73 & 35,06 & 2,42 & 16,06 & 30,00 \\
\hline
\end{tabular}

${ }^{(1)}$ Médias seguidas de letras distintas, nas colunas, diferem pelo teste de Tukey, a 5\% de probabilidade. ANA, ácido naftaleno acético; GA 3 , ácido giberélico.

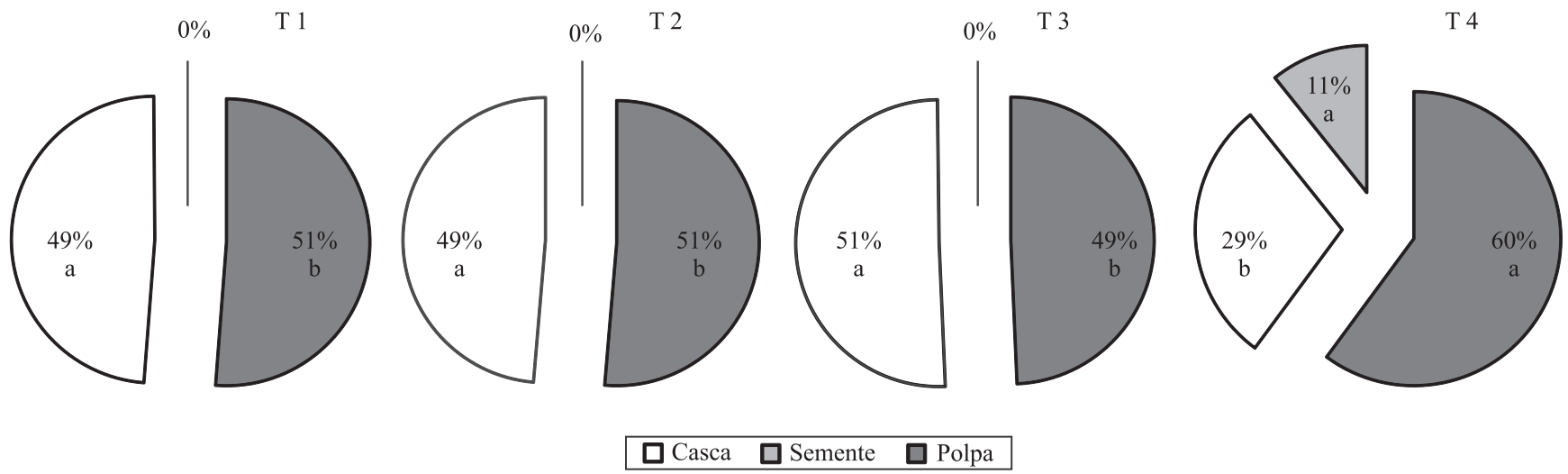

Figura 4. Percentagem de polpa, casca e semente de frutos de atemoia 'Gefner', após a aplicação dos seguintes reguladores de crescimento: T1, ANA a $450 \mathrm{mg} \mathrm{L}^{-1}+\mathrm{GA}_{3}$ a $1.000 \mathrm{mg} \mathrm{L}^{-1}$; T2, promalina a $100 \mathrm{mg} \mathrm{L}^{-1}+\mathrm{GA}_{3}$ a $1.000 \mathrm{mg} \mathrm{L}^{-1}$; T3, GA 3 $1.000 \mathrm{mg} \mathrm{L}^{-1}$; e T4, polinização artificial, em Homestead, Flórida, EUA. ANA, ácido naftaleno acético; GA 3 , ácido giberélico. ${ }^{(1)}$ Médias seguidas de letras distintas, nas mesmas características, diferem pelo teste de Tukey, a 5\% de probabilidade. Coeficientes de variação: polpa, 5,12\%; casca, 5,76\%; e semente, $27,15 \%$. 
irregulares, também foram obtidos por Pereira et al. (2014), ao aplicar $1.000 \mathrm{mg} \mathrm{L}^{-1}$ de $\mathrm{GA}_{3}$, na região semiárida do Norte de Minas Gerais, o que mostra a necessidade de mais estudos sobre o tema.

Os frutos produzidos pela polinização artificial apresentaram maior firmeza do que os advindos da aplicação dos reguladores de crescimento (Tabela 2). Isso pode estar associado à presença de sementes em frutos oriundos de polinização, que foram mais consistentes, inclusive com menor perda de massa de matéria fresca, ao longo do amadurecimento, conforme mostrado anteriormente (Tabela 1). Os frutos provenientes da polinização artificial também apresentaram maior rendimento de polpa, que foi $10 \%$ superior em relação aos advindos da aplicação de reguladores de crescimento (Figura 4). Possivelmente, isso ocorreu graças ao maior desenvolvimento da polpa, que envolve cada semente nos frutos polinizados. Deve-se destacar que os frutos não polinizados, com cerca de $50 \%$ de polpa em média, podem ser considerados bastante viáveis do ponto de vista comercial, já que, em razão da ausência de sementes, o consumo destes frutos é facilitado.

Entre os itens analisados pelo consumidor, no momento da aquisição da maioria dos frutos, podemse destacar as características de uniformidade, firmeza, coloração, tamanho, maior quantidade de polpa, menor número de sementes e sabor. Frutos de atemoia com sementes e com massa de matéria fresca inferior a $300 \mathrm{~g}$ são considerados pequenos para a comercialização e recebem menor preço de mercado (Melo et al., 2002). A produção de frutos menores, mas com boa proporção de polpa e elevado teor de sólidos solúveis, além da ausência de sementes, provenientes da aplicação do $\mathrm{GA}_{3}$, associado ou não ao ANA ou à promalina, mostra o grande potencial para comercialização dos frutos de atemoia.

\section{Conclusões}

1. A aplicação de ácido naftaleno acético, associado com ácido giberélico, a flores de atemoia 'Gefner', proporciona elevada frutificação efetiva, semelhante à polinização artificial.

2. O uso de ácido giberélico, associado ou não ao ácido naftaleno acético ou à promalina, resulta na produção de frutos de atemoia 'Gefner' mais tardios, menores e menos consistentes do que os advindos da polinização artificial, porém, com boa proporção de polpa, excelente teor de sólidos solúveis e, principalmente, sem sementes.

\section{Agradecimentos}

À Fundação de Amparo à Pesquisa do Estado de Minas Gerais (Fapemig) e à Coordenação de Aperfeiçoamento de Pessoal de Nível Superior (Capes), pela concessão de bolsas.

\section{Referências}

BETTIOL NETO, J.E.; DEL NERO, M.; KAVATI, R.; PINTO-MAGLIO, C.A.F. Viabilidade e conservação de pólen de três anonas comerciais. Bragantia, v.68, p.825-837, 2009. DOI: 10.1590/S0006-87052009000400002.

CASANOVA, L.; CASANOVA, R.; MORET, A.; AUGUSTI, M. The application of gibberellic acid increases berry size of 'Emperatriz' seedless grape. Spanish Journal of Agricultural Research, v.7, p.919-927, 2009. DOI: 10.5424/sjar/2009074-1105.

CRANE, J.H.; BALERDI, C.F.; MAGUIRE, I. Sugar apple growing in the Florida home landscape. Gainesville: University of Florida, 2005. 9p.

DORCEY, E.; URBEZ, C.; BLAZQUEZ, M.A.; CARBONELL, J.; PEREZ-AMADOR, A. Fertilization-dependent auxin response in ovules triggers fruit development through modulation of gibberellin metabolism in Arabidopsis. Plant Journal, v.58, p.318-332, 2009. DOI: 10.1111/j.1365-313X.2008.03781.x.

FERREIRA, D.F. Sisvar: um programa para análises e ensino de estatística. Revista Symposium, v.6, p.36-41, 2008.

GOURGET, B.; VAN HEUSDEN, A.W.; LINDHOUT, P. Parthenocarpic fruit development in tomato. Plant Biology, v.7, p.131-139, 2005. DOI: 10.1055/s-2005-837494.

JONG, M. de; MARIANI, C.; VRIEZEN, W.H. The role of auxin and gibberellin in tomato fruit set. Journal of Experimental Botany, v.60, p.1523-1532, 2009. DOI: 10.1093/jxb/erp094.

KIHARA, H. Triploid watermelon. Proceedings of the American Society for Horticultural Science, v.58, p.217-230, 1951.

KOURA, S.; HASEGAWA, K.; YAMAMOTO, Y.; YONEMOTO, Y. Fruit set and fruit growth of seedless cherimoya (Annona cherimola Mill.) induced by $\mathrm{GA}_{3}$ under greenhouse cultivation in Japan. Acta Horticulturae, v.653, p.63-66, 2004.

LORA, J.; HORMAZA, J.I.; HERRERO, M.; GASSER, C.S. Seedless fruits and the disruption of a conserved genetic pathway in angiosperm ovule development. Proceedings of the National Academy of Sciences of the United States of America, v.108, p.5461-5465, 2011. DOI: 10.1073/pnas.1014514108.

MAESTRELLI, A.; LO SCALZO, R.; ROTINO, G.L.; ACCIARRI, N.; SPENA, A.; VITELLI, G.; BERTOLO, G. Freezing effect on some quality parameters of transgenic parthenocarpic eggplants. Journal of Food Engineering, v.56, p.285-287, 2003. DOI: 10.1016/S0260-8774(02)00270-4. 
MELO, M.R.; POMMER, C.V.; KAVATI, R. Polinização artificial da atemóia com diversas fontes de pólen comparada com a natural. Bragantia, v.61, p.231-236, 2002. DOI: 10.1590/ S0006-87052002000300004.

MENDES, H.T.A.; COSTA, M.R.; NIETSCHE, S.; OLIVEIRA, J.A.A.; PEREIRA, M.C.T. Pollen grain germination and fruit set in 'Brazilian seedless' sugar apple (Annona squamosa L). Crop Breeding and Applied Biotechnology, v.12, p.277-280, 2012.

MOREIRA, E.R.; BOLIANI, A.C.; SANTOS, P.C. dos; CORRÊA, L. de S.; MARIANO, F.A. de C.; ATTÍLIO, L.B. Efeito do ácido giberélico e thidiazuron na qualidade de cachos e bagas de uva 'Niagara Rosada'. Tecnologia e Ciência Agropecuária, v.4, p.17-23, 2010.

MOTA FILHO, V.J.G.; PEREIRA, M.C.T.; NIETSCHE, S.; GUIMARÃES, J.F.R.; MOREIRA, G.B.R.; FERNANDES, T.P. Uso de fitorreguladores no desenvolvimento de frutos na atemoia (Annona cherimola x A. squamosa cv. Gefner). Revista Ceres, v.59, p.636-645, 2012. DOI: 10.1590/S0034-737X2012000500009.

NITSCH, J.P. Plant hormones in the development of fruits. The Quarterly Review of Biology, v.27, p.33-57, 1952. DOI: 10.1086/398643.

OZGA, J.A.; REINECKE, D.M. Interaction of 4-chloroindole3-acetic acid and gibberellins in early pea fruit development. Plant Growth Regulation, v.27, p.33-38, 1999. DOI: 10.1023/A:1006151401685.

PANDOLFINI, T. Seedless fruit production by hormonal regulation of fruit set. Nutrients, v.1, p.168-177, 2009. DOI: 10.3390/nu1020168.

PEREIRA, F.M.; KAVATI, R. Contribuição da pesquisa científica brasileira no desenvolvimento de algumas frutíferas de clima subtropical. Revista Brasileira de Fruticultura, p.92-108, 2011. Volume especial.

PEREIRA, M.C.T.; NIETSCHE, S.; COSTA, M.R.; CRANE, J.H.; CORSATO, C.D.A.; MIZOBUTS, E.H. Anonáceas: pinha, atemoia e graviola. Informe Agropecuário, v.32, p.26-34, 2011.

PEREIRA, M.C.T.; NIETSCHE, S.; SANTOS, F.S.; XAVIER, A.A.; CUNHA, L.M.V.; NUNES, C.F.; SANTOS, F.A. Efeito de horários de polinização artificial no pegamento e qualidade de frutos de pinha (Annona squamosa L.). Revista Brasileira de Fruticultura, v.25, p.203-205, 2003. DOI: 10.1590/ S0100-29452003000200004.

PEREIRA, M.C.T.; SANTOS, R.K.A.; NIETSCHE, S.; MIZOBUTSI, G.P.; SANTOS, E.F. dos. Doses de ácido giberélico na frutificação efetiva e qualidade de frutos de atemoia 'Gefner'. Revista Brasileira de Fruticultura, v.36, p.184-191, 2014. DOI: 10.1590/S0100-29452014000500022.

PIRES, E.J.P.; BOTELHO, R.V.; TERRA, M.M. Efeitos do CPPU e do ácido giberélico nas características dos cachos da uva de mesa 'Centennial Seedless'. Ciência e Agrotecnologia, v.27, p.305-311, 2003. DOI: 10.1590/S1413-70542003000200008.

RICHARDS, F.C. The quantitative analysis of plant growth. In: STEWARD, F.C. Plant physiology: a treatise. New York: Academic Press, 1969. p.3-76.

SAAVEDRA, E. Set and growth of Annona cherimola Mill. fruit obtained by hand-pollination and chemical treatments. Journal of the American Society for Horticultural Science, v.104, p.668-673, 1979.

SCHWABE, W.W.; MILLS, J.J. Hormones and parthenocarpic fruit set: a literature survey. Horticultural Abstracts, v.51, p.661-668, 1981.

SERRANI, J.C.; SANJUÁN, R.; RUIZ-RIVERO, O.; FOS, M.; GARCÍA-MARTINEZ, J.L. Gibberellin regulation of fruit set and growth in tomato. Plant Physiology, v.145, p.246-257, 2007. DOI: 10.1104/pp.107.098335.

SRIVASTAVA, A.; HANDA, A.K. Hormonal regulation of tomato fruit development: a molecular perspective. Journal of Plant Growth Regulation, v.24, p.67-82, 2005. DOI: 10.1007/ s00344-005-0015-0.

TECCHIO, M.A.; LEONEL, S.; CAMILI, E.C.; MOREIRA, G.C.; PAIOLI-PIRES, E.J.; RODRIGUES, J.D. Uso de bioestimulante na videira 'Niagara Rosada'. Ciência e Agrotecnologia, v.30, p.1236-1240, 2006. DOI: 10.1590/S1413-70542006000600030.

VARGA, A.; BRUINSMA, J. Tomato. In: MONSELISE, S.P. (Ed.). CRC handbook of fruit set and development. Boca Raton: CRC Press, 1986. p.461-480.

YONEMOTO, Y.; HIGUCHI, H.; TOMITA, E. Possibility of producing seedless fruits in 11 cultivars of cherimoya by GA3 application. Japanese Journal of Tropical Agriculture, v.44, p.72-74, 2000.

Recebido em 5 de dezembro de 2013 e aprovado em 17 de março de 2014 